\title{
GENERATION OF FEASIBLE TEST SEQUENCES FOR EFSM MODELS
}

\author{
Ali Y. Duale and M. Ümit Uyar \\ Electrical Engineering Department \\ The City College of the City University of New York \\ [duale, umit]@ee-mail.engr.ccny.cuny.edu
}

\begin{abstract}
A method that enables the generation of realizable test sequences from a class of EFSMs is presented. If the interdependencies among the variables used in the actions and the conditions of EFSMs are not considered during test generation, the test sequences may be unrealizable in a test laboratory. Algorithms for the detection and elimination of inconsistencies from the EFSM models are presented. Once inconsistencies are eliminated, realizable test sequences can be generated from the resulting consistent EFSM by using the methods available for FSM models.
\end{abstract}

Keywords: conformance testing, EFSM, Estelle, FSM, VHDL.

\section{INTRODUCTION}

The aim of conformance testing is to detect errors and increase the level of confidence on an implementation. A major challenge in conformance testing of complex communication systems is the development of methods that automatically generate test sequences with minimum lengths while maximizing the fault coverage. The automation of test generation from the extended finitestate machines (EFSMs) is mainly impaired by the existence of inconsistencies among the variables used in the actions and conditions of the EFSM models.

\footnotetext{
*Prepared through collaborative participation in the Advanced Telecommunications \& Information Distribution Research Program (ATIRP) Consortium sponsored by the U.S Army Research Laboratory under the Federated Laboratory Program, Cooperative Agreement DAAL01-96-2-000-2. The US Government is authorized to reproduce and distribute reprints for Government purposes notwithstanding any copyright notation thereon.
} 
The specifications written in formal description languages such as VHDL and Estelle are typically modeled as EFSMs.

Conformance test generation from the finite-state machine (FSM) or labeled transition system (LTS) models has been an active research area $[2,6,9,10,11$, $13,17,18,19]$. However, generating feasible test sequences from the EFSMs still remains as an open research problem. Although several researchers made significant contributions towards testing EFSM models $[3,4,12,14,15,16,19$, 20], the inclusion of infeasible paths in the test sequences may be inevitable as long as the underlying models are EFSMs. Without a proper analysis of the interdependencies among the variables used in the actions and conditions of the EFSMs, considerable effort may be wasted on test generation since infeasible portions of these test sequences will be discarded later. The method presented in this paper enables the generation of only feasible test sequences from a class of EFSMs. It is assumed that the specification consists of a single process with linear actions and conditions. It is also assumed that pointers, recursive functions, and syntactically endless loops are not present in the specification.

The methodology presented here first considers the inconsistencies caused by the actions of the EFSM (if any), and proceeds with the elimination of inconsistencies among the conditions of the EFSM (if any).

The examples presented in this paper are based on VHDL. However, the algorithms are applicable to all EFSMs with the aforementioned properties. Currently, these algorithms are being applied to solve the conflicting timers problem, which arises when a protocol has multiple timers running concurrently. Due to the conflicting timers, a test sequence of a protocol such as the Estelle specification of the MIL STD 188-220 [8] may be interrupted by unexpected timeouts. Preliminary results show that generating test sequences for the MIL-STD 188-220 after eliminating the timer inconsistencies significantly improves the test coverage by including more transitions into the test sequences without timer interruptions [7].

The rest of the paper is organized as follows. Section 2. provides background information. Analysis of inconsistencies in EFSM models is presented in Section 3.. An example is worked out in Section 4.. Concluding remarks are given in Section 5..

\section{PRELIMINARIES}

The control structure of a communication protocol can be modeled as an FSM whereas the data portion of a protocol is usually modeled as an EFSM.

An $F S M=\langle S, I, O, \delta, \lambda\rangle$ where $S, I, O, \delta$, and $\lambda$ are a finite set of states, a finite set of inputs, a finite set of outputs, a state transition function such that $\delta: S \mathrm{x} I \rightarrow S$, and an output function such that $\lambda: S \times I \rightarrow O$, respectively. An $E F S M=\langle S, I, O, X, P, B, T\rangle$ where $S, I$, and $O$ are defined as in an FSM; 
$X, P, B$, and $T$ are a set of variables, a set of parameters associated with the input set, a set of Boolean expressions which reference to a memory location, and a set of actions of the EFSM that consists of spontaneous transitions ( $S T$ ) and input transitions $(I T)$, respectively.

Both FSMs and EFSMs can be represented as directed graphs. A graph $G(V, E)$ is a structure defined by two nonempty sets, $V$ and $E$. The elements of $V$ and $E$ are called nodes (or vertices) and edges, respectively. A pair of ordered nodes, $\left(v_{i}, v_{j}\right)$, of a directed graph describes an edge $e_{k} \in E$ which leaves $v_{i}$ and terminates on $v_{j}$. The nodes $v_{i}$ and $v_{j}$ are called the head and the tail nodes of $e_{k}$, respectively.

A path $(P)$ in $G(V, E)$ is a non-null sequence of consecutive edges. If the starting and ending nodes of a path (i.e., $v_{1}$ and $v_{r}$ ) are the same, the path forms a loop. For simplicity, throughout this paper, the term loop refers to the VHDL while/for constructs, rather than the ordinary loops. (The algorithms are applicable to both graphs with the ordinary loops and while/for loop constructs.) A node in $G$ is assumed to be identified as the initial node from which all other nodes are reachable, and vice versa. $G^{\prime}\left(V^{\prime}, E^{\prime}\right)$ is called a subgraph of $G(V, E)$ (expressed as $G^{\prime}\left(V^{\prime}, E^{\prime}\right) \subseteq G(V, E)$ ) if:

$$
V^{\prime} \subseteq V \text { and } E^{\prime} \subseteq E: \forall e_{i}^{\prime} \in E^{\prime} \operatorname{tail}\left(e_{i}^{\prime}\right), \operatorname{head}\left(e_{i}^{\prime}\right) \in V^{\prime}
$$

\section{INCONSISTENCIES}

One of the major differences between the FSMs and EFSMs stems from the memory (i.e., the variables used in conditions) associated with the EFSMs. Unlike FSM graphs, the traversal of an edge $e_{k}=\left(v_{i}, v_{j}\right)$ of an EFSM graph mainly depends upon the condition and action variables of the edges in the paths leading to $v_{i}$. In an EFSM graph, the traversal of an edge leaving a node $v_{i}$ may not be possible due to the conflicting conditions of the path(s) from $v_{0}$ to $v_{i}$, whereas for an FSM graph all paths are feasible. The complexity of testing EFSM models increases when one or more variables used in the edge conditions can assume multiple values at the same node.

A comprehensive analysis of the inconsistencies among the actions and conditions of the EFSM models is given in the subsequent sections. To improve readability, a simple example is chosen in this paper; however, the example is designed to depict the inconsistencies commonly present in real-life communication protocol specifications (e.g., MIL-STD 188-220).

Let us represent the condition of an edge as:

$$
a_{00} x_{0}+a_{01} x_{1}+\ldots+a_{0(m-1)} x_{m-1}<o p>d
$$

and the action of an edge as:

$$
x_{i}=a_{01} x_{0}+a_{11} x_{1}+\ldots+a_{1(m-1)} x_{m-1}+d
$$


where $m, o p$, and $d$ are the number of variables, an operator, and a constant, respectively.

Definition 1 Condition inconsistency: If there is no solution for the set of equations formed by the accumulated conditions of the edges of a sub-path $e_{1} \cdot e_{2} \cdot \ldots \cdot e_{i}$ and an edge $e_{j}$, where head $\left(e_{j}\right)$ can be reached from tail $\left(e_{i}\right)$ or head $\left(e_{j}\right)=\operatorname{tail}\left(e_{i}\right)$, then $e_{i}$ and $e_{j}$ are said to have a condition inconsistency.

Definition 2 Action inconsistency: If there is no solution for the set of equations formed by the actions of an edge $e_{i}$ and the condition of another edge $e_{j}$, where head $\left(e_{j}\right)$ can be reached from tail $\left(e_{i}\right)$ or head $\left(e_{j}\right)=\operatorname{tail}\left(e_{i}\right)$, then the two edges of $e_{i}$ and $e_{j}$ are said to have an action inconsistency.

Definition 3 Consistent EFSM: An EFSM which is free of both action and condition inconsistencies is called a consistent EFSM.

In a consistent EFSM, the variables used in the conditions and actions do not impose any restrictions over the paths. Hence, all paths of the graph representation of a consistent EFSM are feasible.

In this paper, a depth-first (DF) and a modified breadth-first (MBF) graph traversals are used to detect inconsistencies together with symbolic execution and linear programming. Note that symbolic execution is utilized as a tool to detect inconsistencies, not as a test generation tool. For the inconsistency elimination, a graph splitting technique based on symbolic execution and linear programming [1] is introduced.

Once the inconsistencies are eliminated from an EFSM graph, realizable test sequences can be automatically generated from the resulting consistent EFSM by using the test generation methods available for FSM models (see for example [2] - [4], [6], [10] - [20]).

\subsection{Definitions}

Let us introduce the terms used throughout the paper as:

- $V_{v_{i}}^{\text {reachable }} \subseteq V$ : set of nodes reachable from $v_{i}$ without touching $v_{0}$, the initial node.

- $E_{v_{i}}^{\text {reachable }} \subseteq E$ : set of outgoing edges of $V_{v_{i}}^{\text {reachable }}$.

- $E_{v_{i}}^{\text {out }} \subseteq E$ : set of outgoing edges of $v_{i}$.

- $E_{e_{i} \sim e_{j}}^{\text {out }} \subseteq E$ : set of edges in the paths between $\operatorname{tail}\left(e_{i}\right)$ and $h e a d\left(e_{j}\right)$.

- $E_{v_{i}}^{i n} \subseteq E$ : set of incoming edges of $v_{i}$.

- $E_{v_{i}}^{i n(T)} \subseteq E_{v_{i}}^{i n}$ : set of incoming edges of $v_{i}$ that have already been traversed.

- $E_{v_{i}}^{i n(N T)}=E_{v_{i}}^{i n}-E_{v_{i}}^{i n(T)}$ : set of incoming edges of $v_{i}$ that have not traversed.

- $L_{o o p} v_{v_{i}} \in V$ : node $v_{i}$ is a loop entry/exit node. 
- $V^{\text {Loop }_{v_{i}}} \subset V$ : set of nodes that constitute the loop body whose entry/exit node is $v_{i}$.

- $E_{L_{o o p} p_{i}}^{\text {exit }} \subset E$ : set of outgoing edges of $L_{o o p} p_{v_{i}}$ whose tail nodes are in the set $V_{\text {Loop }_{i}}^{\text {reachable }}-V^{\text {Loop }_{v_{i}} \text {. }}$

- $V A R=\left\{v^{2} r_{1}, v_{a r_{2}}, \cdots, v a r_{m}\right\}$ : set of variables used in the edge conditions and actions of $G(V, E)$.

- $V A R_{v_{i}}^{\text {con-used }} \subseteq V A R$ : set of variables used in the conditions of outgoing edges of $V_{v_{i}}^{\text {reachable }} \cup\left\{v_{i}\right\}$.

- $V A R_{v_{i}}^{\text {dif-modified }} \subseteq V A R$ : set of variables modified differently in the paths leading to $v_{i}$.

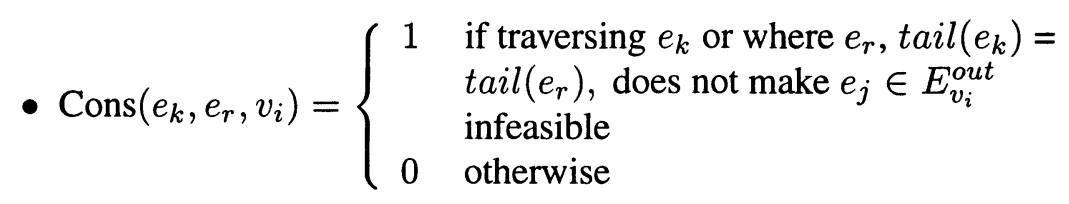

For simplicity, the notations $v_{i(s)}$ and $v_{i}$ as well as $e_{i(s)}$ and $e_{i}$ will be used interchangeably where appropriate.

\subsection{Detection and Removal of Inconsistencies}

The algorithms presented in this paper eliminate the inconsistencies by creating new nodes and edges, which increases the size of the original graph. However, these new nodes and edges are created only when necessary. Therefore, although the well-known state explosion cannot be avoided for all EFSMs, the unnecessary growth of the state space is prevented. For the cases where the state explosion is unavoidable, the size of the new graph is constantly monitored as the algorithms eliminate the inconsistencies.

3.2.1 Action Inconsistencies. Variables modified in the paths leading to a node $v_{i}$ may cause action inconsistencies with the condition of another edge $e_{r}$, where head $\left(e_{r}\right) \in V_{v_{i}}^{\text {reachable }}$. Therefore, the effects of the variables modified differently by the actions of the paths leading to a node $v_{i}$ on the conditions of the edges reachable from $v_{i}$ need to be analyzed.

In general, the effects of edge actions on variables (i.e., variable modifications) can be represented as matrices. For an EFSM graph with $m$ variables, $v a r_{1}, v a r_{2}, \ldots, v r_{m}$, a pair of matrices $A(m \times m)$ and $\tilde{B}(m \times 1)$ called the modification matrix and the modification vector, respectively, are defined.

The accumulated effects of the actions in the paths leading to a node $v_{i}$ can be represented by a set of Action Update Matrix pairs defined as:

$$
\operatorname{AUM}\left(v_{i}, J\right)=\left\{A_{v_{i}, 0}, \tilde{B}_{v_{i}, 0}, A_{v_{i}, 1}, \tilde{B}_{v_{i}, 1}, \cdots, A_{v_{i}, J-1}, \tilde{B}_{v_{i}, J-1}\right\}
$$


where $A_{v_{i}, k}, \tilde{B}_{v_{i}, k}$, and $J$ are the $k^{\text {th }}$ modification matrix, $k^{\text {th }}$ modification vector $(0 \leq k<J)$, and the number of AUM pairs associated with $v_{i}$, respectively. The symbolic values of a variable $v a r_{r}$ are represented in the $r^{\text {th }}$ rows in $\operatorname{AUM}\left(v_{i}, J\right)$. Only one AUM pair, where $A$ and $\tilde{B}$ are initialized to the identity matrix and to a zero vector, respectively, is created for the initial node.

The number of AUM pairs associated with $v_{i}$ solely depends on the number of different ways in which the actions of the edges leading to $v_{i}$ modify variables. If the overall variable modifications of the actions of any two paths leading to $v_{i}$ are the same, only one AUM pair is sufficient to account for the effects of the actions in the two paths. Therefore, only unique AUM pairs are associated with $v_{i}$.

In general, after traversing an edge $e_{k}=\left(v_{i}, v_{j}\right)$, new AUM pairs are formed for $v_{j}$ by applying the actions of $e_{k}$ to the $\operatorname{AUM}\left(v_{i}, J\right)$. Depending upon the net modification on $\operatorname{AUM}\left(v_{i}, J\right)$ by the actions of $e_{k}$, the number of new AUM pairs formed for $v_{j}$ may be less than or equal to that of $v_{i}$.

3.2.1.1 Detection of Action Inconsistencies. In this paper, a two-phase MBF graph traversal is designed to handle the detection of the action inconsistencies. The two phases of the MBF graph traversal will be referred to as $\mathrm{P} 1-\mathrm{MBF}$ and P2-MBF. Phase one of the MBF graph traversal, P1-MBF, can be viewed as the main graph traversal from which the P2-MBF may be invoked multiple times.

In the P1-MBF graph traversal, certain restrictions are imposed on the traversal of some edges as described below:

- The traversal of the edges of $E_{L^{e o p} p_{v_{i}}}^{\text {xit }}$ is postponed until the edges of $\left(E_{\text {Loop }_{v_{i}}}^{\text {out }}-E_{\text {Loop }_{v_{i}}}^{\text {exit }}\right.$ ) are traversed.

- The traversal of the outgoing edges of $v_{j} \in V^{\text {Loop }_{v_{i}}}$ is postponed until the edges of $\left(E_{\text {Loop }_{v_{i}}}^{\text {in }}-\left\{e_{m}=\left(v_{x}, v_{y}\right): v_{x} \in V^{\text {Loop }_{v_{i}}}\right\}\right)$ are traversed.

- The analysis of a loop (except for nested loops) whose entry/exit node is $\operatorname{loop}_{v_{i}}$ is avoided if there is a non-traversed edge $e_{m}=\left(v_{x}, v_{y}\right)$,

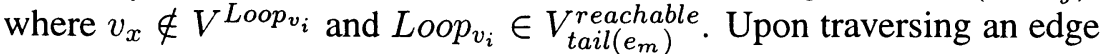
$e_{k}=\left(v_{x}\right.$, Loop $\left._{v_{i}}\right)$, it is checked if the following condition is true:

$$
\begin{aligned}
& \exists e_{r}=\left(v_{y}, v_{w}\right) \in E_{v_{w}}^{\text {in(NT) }} \text { such that } \\
& \left(\operatorname{Loop}_{v_{i}} \in V_{v_{w}}^{\text {reachable }} \wedge \operatorname{Loop}_{v_{i}} \notin V^{\text {Loop }_{v_{w}}} \wedge \operatorname{head}\left(e_{r}\right) \notin V^{\text {Loop }_{v_{w}}}\right)
\end{aligned}
$$

If statement (1) is true, the traversal of the edges of $E_{L_{o o p}}^{\text {out }}$ is postponed. As can be seen from (1), for nested loops, an inner loop is analyzed before traversing certain incoming edges of the outer loop entry/exit node. 
In the P1-MBF graph traversal, upon traversing an edge $e_{k}=\left(v_{x}, v_{y}\right)$, it is checked if an action inconsistency between the edges in the paths leading to $v_{y}$ and an edge $e_{r}=\left(v_{w}, v_{z}\right)$ reachable from $v_{y}$ exists. The detection of action inconsistencies starts with checking the number of AUM pairs associated with $v_{y}$. If there is more than one, the effects of $\operatorname{AUM}\left(v_{y}, J\right)$ on the conditions of

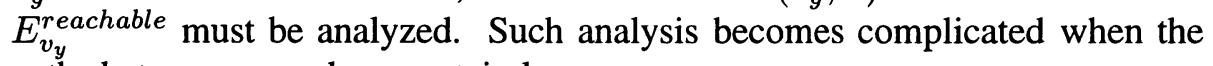
paths between $v_{y}$ and $v_{w}$ contain loops.

When a node with multiple AUM pairs is visited by using P1-MBF, the $\mathrm{P} 2-\mathrm{MBF}$ graph traversal is initiated. If there is a loop in the path(s) between $v_{y}$ and tail $\left(e_{i}\right), \mathrm{P} 2-\mathrm{MBF}$ aims to postpone the analysis of the effects of the $\operatorname{AUM}\left(v_{y}, J\right)$ on the conditions of $e_{i} \in E_{v_{y}}^{r e a c h a b l e}$ until this loop is completely analyzed. To cope with the difficulty of analyzing the effects of the $\operatorname{AUM}\left(v_{y}, J\right)$ on the conditions of $E_{v_{y}}^{\text {reachable }}, \mathrm{P} 2-\mathrm{MBF}$ allows the traversal of each edge of a loop body at most once. In the P2-MBF graph traversal, edges of $E_{L o o p_{v_{i}}}^{\text {exit }}$ are not traversed until the loop whose entry/exit node is $L_{o o p} p_{i}$ is completely analyzed by P1-MBF. In addition, unlike P1-MBF, the loop iterations are not advanced during the $\mathrm{P} 2-\mathrm{MBF}$ graph traversal.

In P2-MBF, the traversal of a given path terminates if at least one of the following statements is true:

- an action inconsistency is detected

- the path contains a loop entry/exit node $\operatorname{Loop}_{v_{i}}$ where

- all edges in $\left(E_{\text {Loop }_{v_{i}}}^{\text {out }}-E_{\text {Loop }_{v_{i}}}^{\text {exit }}\right)$ are traversed or

- another entry/exit node $\operatorname{Loop}_{v_{j}} \in V^{\text {Loop }_{v_{i}}}$ is visited by traversing an edge $e_{k}=\left(v_{x}, \operatorname{Loop}_{v_{j}}\right)$

The action inconsistency detection algorithm is given in Figure 1, where the first while loop is $\mathrm{P} 1-\mathrm{MBF}$ and the if statement within the second while loop implicitly represents $\mathrm{P} 2-\mathrm{MBF}$. The algorithm stops after finding the first action inconsistency. If there is no action inconsistency, the algorithm stops upon completing the traversal of the graph in the MBF manner.

3.2.1.2 Elimination of Action Inconsistencies. The algorithm Figure 2 eliminates an action inconsistency between $e_{k}=\left(v_{x}, v_{y}\right)$ and $e_{r}=\left(v_{w}, v_{z}\right)$ of a loop-free graph by placing them into two separate subgraphs to prevent these two edges from being included in the same path. The two subgraphs are formed by splitting nodes and edges of $\left(\left\{v_{y}\right\} \cup V_{v_{y}}^{\text {reachable }}\right)$ and $\left(E_{v_{y}}^{\text {out }} \cup E_{v_{y}}^{\text {reachable }}\right)$ such that each subgraph contains either $e_{k}$ or $e_{r}$ but not both. When a node $v_{i(s)}$ is split, the new duplicate of $v_{i}$ is denoted as $v_{i\left(s^{*}\right)}$, where $s^{*}$ represents the number of times that $v_{i(s)}$ is split. Similarly, the duplicate of an edge $e_{i}$ is denoted in the same manner (i.e., $\left.e_{i\left(s^{*}\right)}\right)$.

Only the edges of $E_{v_{y}}^{i n}=\left(E_{v_{y}}^{T} \cup E_{v_{y}}^{N T}\right)$ that do not conflict with the edges in $E_{v_{w}}^{o u t}$ are duplicated during the splitting. However, it is not apparent if placing 


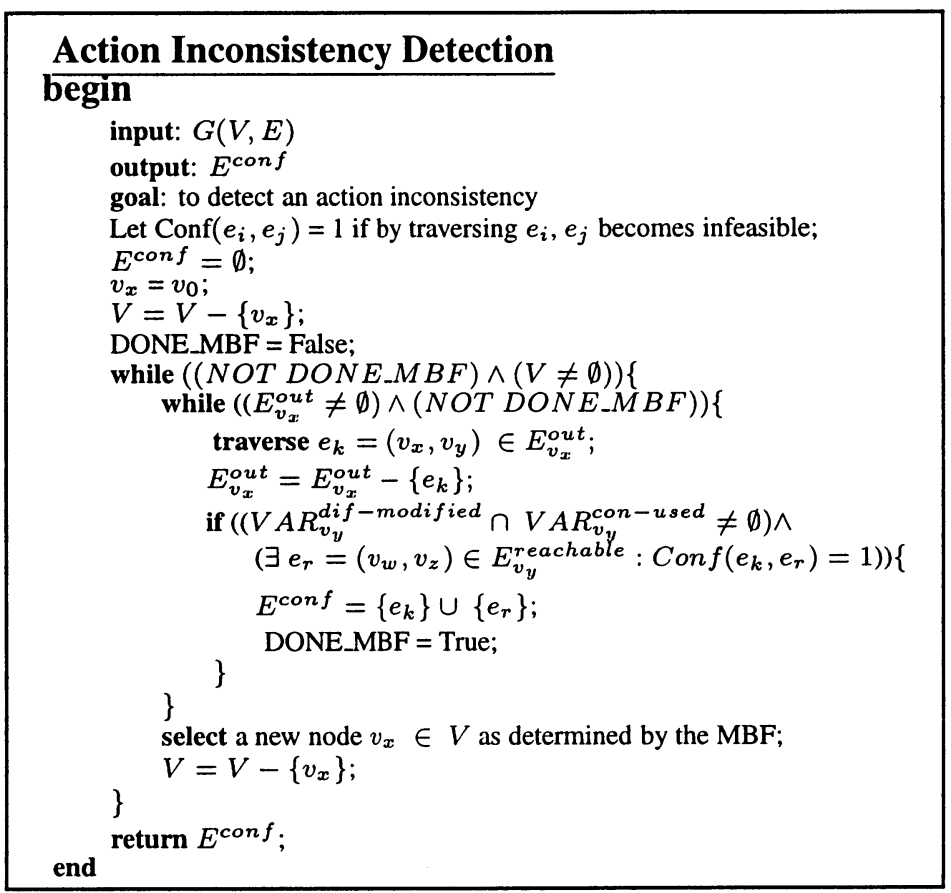

Figure 1. Action Inconsistency detection algorithm.

copies of the edges in $E_{v_{y}}^{N T}$ in the same subgraph with $e_{k}=\left(v_{x}, v_{y}\right)$ will cause action inconsistencies. Copies of such edges are temporarily included in the same subgraph with $e_{k}$.

The effects of the actions of $E_{v_{w}}^{\text {depend }}$ on the outgoing edges of $v_{w} \in$ $V_{\text {tail }\left(e_{k}\right)}^{\text {reachable }}$ must be analyzed later when each edge in $E_{v_{w}}^{\text {depend }}$ is traversed, where $E_{v_{w}}^{\text {depend }}$ is the set of edges temporarily included in the subgraph containing $e_{k}$. When an edge $e_{i\left(s^{*}\right)}^{\prime} \in E_{v_{w\left(s^{*}\right)}^{\prime}}^{\text {depend }}$ is traversed, it is checked if the actions of $e_{i\left(s^{*}\right)}^{\prime}$ are inconsistent with the conditions of the outgoing edges of $v_{w\left(s^{*}\right)}^{\prime}$. If they are, $e_{i\left(s^{*}\right)}^{\prime}$ is removed from the graph. Recall that $v_{w\left(s^{*}\right)}^{\prime}$ is one of the copies of $v_{w(s)}$ whose outgoing edges conflicted with $e_{k(s)}$.

The graph splitting is slightly different when an action inconsistent is detected by traversing $e_{k}=\left(v_{x}, v_{y}\right)$, where $v_{y}$ is a loop entry/exit node, as will be described later.

Since the graph topology changes with the creation of the new edges and nodes, the action inconsistency detection algorithm should be re-invoked after each graph split. As defined in Section 3.1, a path from $v_{i}$ to $v_{j}$ cannot include $v_{0}$. Hence, the inconsistency removal algorithm does not split $v_{0}$.

As for the loop-free graphs, the action inconsistency detection is performed by using the two-phase MBF graph traversal with P1-MBF and P2-MBF. For 


\section{Action Inconsistency Elimination}

input: $e_{k}=\left(v_{x}, v_{y}\right)$ and $e_{r}=\left(v_{w}, v_{z}\right)$, where an action inconsistency exists between $e_{k}$ and $e_{r}$

goal: to eliminate the inconsistency between $e_{k}$ and $e_{r}$ by creating begin two subgraphs such that each subgraph contains either $e_{k}$ or $e_{r}$, but not both

Split $v_{y(s)}$ into two nodes as: $v_{y(s)}^{\prime}$ and $v_{y\left(s^{*}\right)}^{\prime}$;

Split $\forall v_{i(s)} \in V_{v_{y(s)}^{r e a c h a b l e}}^{\text {into two nodes as: }}$

$v_{i(s)}^{\prime} \in V_{v_{y(s)}^{\prime}}^{\text {reachable }}$ and $v_{i\left(s^{*}\right)}^{\prime} \in V_{v_{y\left(s^{*}\right)}^{\text {reachable }}}^{\text {rea }}$

Split $\forall e_{m(s)}=\left(v_{i(s)}, v_{j(s)} ; L_{e_{m(s)}}\right) \in E_{v_{y(s)}^{r e a c h a b l e}}$ into two edges as:

$$
\begin{aligned}
& e_{m(s)}^{\prime}=\left(v_{i(s)}^{\prime}, v_{j(s)}^{\prime} ; L_{e_{m(s)}}\right) \in \underset{E^{r e a c h a b l e}}{E_{y(s)}^{\prime}} \text { and } \\
& e_{r\left(s^{*}\right)}^{\prime}=\left(v_{i\left(s^{*}\right)}^{\prime}, v_{j\left(s^{*}\right)}^{\prime} ; L_{e_{m(s)}}\right) \in \underbrace{E^{\prime}}_{v_{y\left(s^{*}\right)}^{r e a c h a b l e}}
\end{aligned}
$$

Split $\forall e_{m(s)}=\left(v_{i(s)}, v_{y(s)} ; L_{e_{m(s)}}\right) \in E_{v_{y(s)}}^{i n(T)}$ such that

$\operatorname{Cons}\left(e_{m(s)}, e_{k(s)}, v_{w(s)}\right)=1$, into two edges as:

$e_{m(s)}^{\prime}=\left(v_{i(s)}, v_{y(s)}^{\prime} ; L_{e_{m(s)}}\right) \in E_{v_{y(s)}^{\prime \prime}}^{i n}$ and

$e_{m\left(s^{*}\right)}^{\prime}=\left(v_{i(s)}, v_{y\left(s^{*}\right)}^{\prime} ; L_{e_{m(s)}}\right) \in E_{E_{y\left(s^{*}\right)}^{i n}}^{i n}$ where $v_{w(s)}$ is the node whose outgoing edges are inconsistent with $e_{k(s)}$;

Create a duplicate of $\forall e_{m(s)}=\left(v_{i(s)}, v_{y(s)} ; L_{e_{m(s)}}\right) \in E_{v_{y(s)}^{i n(T)}}^{i n u c h ~ t h a t ~}$

Cons $\left(e_{m(s)}, e_{k(s)}, v_{w(s)}\right)=0$ as:

$$
e_{m(s)}^{\prime}=\left(v_{i(s)}, v_{y(s)}^{\prime} ; L_{e_{m(s)}}\right) \in E_{v_{y(s)}^{i n}}^{i} ;
$$

Split $\forall e_{m(s)}=\left(v_{i(s)}, v_{y(s)} ; L_{e_{m(s)}}\right) \in E_{v_{y(s)}^{i n(N T)}}^{i n t o ~ t w o ~ e d g e s ~ a s: ~}$

$$
\begin{aligned}
& e_{m(s)}^{\prime}=\left(v_{i(s)}, v_{y(s)}^{\prime}, L_{e_{m(s)}}\right) \in E_{v_{y(s)}^{i n(N T)}}^{E_{(s)}^{\prime}} \text { and } \\
& e_{m\left(s^{*}\right)}^{\prime}=\left(v_{i(s)}, v_{y\left(s^{*}\right)}^{\prime}, L_{e_{m(s)}}\right) \in \underbrace{\prime}_{v_{w\left(s^{*}\right)}^{\text {depend }}} \text { (defined below); }
\end{aligned}
$$

end

Figure 2. Action inconsistency elimination algorithm.

simplicity, only loops with single entry/exit nodes are considered in this paper. Note that syntactically endless loops are not considered in this paper.

If by traversing an edge $e_{k}=\left(v_{x}, v_{y}\right)$, of a graph with loops, an action inconsistency is detected with one of the edges in $E_{v_{y}}^{\text {reachable }}$, then one of the following cases is true:

- Case 1: $v_{x} \notin V^{\text {Loop }_{v_{i}}}$.

- Case 2: $v_{x}, v_{y} \in V^{\text {Loop }_{v_{i}}}$ and $y \neq i$ (i.e., tail $\left(e_{k}\right)$ is not the loop entry/exit node).

- Case 3: $v_{x}, v_{y} \in V^{\text {Loop }_{v_{i}}}$ and $y=i$ (i.e., tail $\left(e_{k}\right)$ is the loop entry/exit node).

The method of graph splitting for Cases 1 and 2 is similar to that of the loopfree graphs except that if $\operatorname{Loop}_{v_{i}}$ is split, a copy of each edge $e_{r} \in E_{\text {Loop }_{v_{i}}}^{\text {out }}$ 
is included in all new subgraphs. For Case 3, the following steps are taken to eliminate action inconsistencies:

- The loop is advanced one iteration by duplicating all nodes of $V_{\text {Loop }_{v_{i}}}^{\text {reachable }}$ and the edges of $E_{v_{y}}^{\text {reachable as: }} V_{v_{y(s)}}^{\text {reachable }}, V_{v_{y\left(s^{*}\right)}}^{\text {reachable }}, E_{v_{y(s)}}^{\text {reachable }}$, and $E_{v_{y\left(s^{*}\right)}}^{\text {reachable }}$, respectively. Since a loop must have only one entry node, $\forall e_{r}=\left(v_{x}, v_{y}\right) \in E_{\text {Loop }_{v_{i}}}^{\text {in }}: v_{x} \notin V^{\text {Loop }_{v_{i}}}$ are not duplicated.

- The AUM pairs of $L_{o o p} v_{i}$ are not updated.

- The tail node of $e_{k(s)}$ is changed to $\operatorname{Loop}_{v_{i\left(s^{*}\right)}^{\prime}}$.

- To determine the exit condition(s) of the loop, the feasibility of the conditions of each edge in $E_{L_{o o p} v_{i(s)}^{\prime}}^{\text {out }}$ is investigated. If the loop exit criterion is satisfied, the conditions of the edges in $\left(E_{L_{o o p}}^{\text {out }},-E_{v_{i(s)}^{\prime}}^{\text {exit }}\right.$,oop $\left.v_{i(s)}^{\prime}\right)$ become

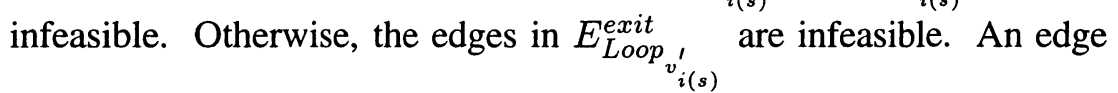
whose condition is found to be infeasible is removed from the graph.

As in the case of loop-free graphs, these steps are repeated after each graph split.

Let us introduce the following definitions for any node $v_{i} \in V$ :

- $\operatorname{AUM}\left(v_{i}, J\right)\left[v_{i} \stackrel{S E}{\sim} v_{x}\right]$ : the resulting $\operatorname{AUM}\left(v_{x}, J\right)$ after applying symbolic execution on $\operatorname{AUM}\left(v_{i}, J\right)$ in the paths between $v_{i}$ and $v_{x} \in V$.

- $E_{v_{i}}^{c o n f\left(e_{x} \in E_{v_{i}}^{i n}, e_{r}\right)} \subseteq E_{v_{i}}^{i n(T)}$ : set of traversed incoming edges of $v_{i}$, where each edge conflicts with $e_{r}$ after obtaining $\operatorname{AUM}\left(v_{i}, J\right)\left[v_{i} \stackrel{S E}{\sim}\right.$ head $\left.\left(e_{r}\right)\right]$.

- $E_{e_{k} \sim e_{r}}^{\text {conf }\left(e_{x} \in E_{e_{k} \sim e_{r}}^{o u t}, e_{r}\right)}$ : set of edges in the paths from tail $\left(e_{k}\right)$ to head $\left(e_{r}\right)$, where each edge conflicts with $e_{r}$ after obtaining

$\operatorname{AUM}\left(\operatorname{tail}\left(e_{k}\right), J\right)\left[\operatorname{tail}\left(e_{k}\right) \stackrel{S E}{\sim} h e a d\left(e_{r}\right)\right]$.

- $E_{v_{i} \sim h e a d\left(e_{r}\right)}^{\text {inf }\left(v_{i}, \text { head }\left(e_{r}\right)\right)}$ set of edges, in the paths between $v_{i}$ and head $\left(e_{r}\right)$, whose conditions are infeasible after obtaining $\operatorname{AUM}\left(v_{i}, J\right)\left[v_{i} \stackrel{S E}{\sim}\right.$ head $\left.\left(e_{r}\right)\right]$.

After an action is detected and eliminated, the resulting graph $G^{\prime}\left(V^{\prime}, E^{\prime}\right)$ is characterized as:

$$
\begin{aligned}
& V^{\prime}=\left(V-V_{v_{y(s)}}^{\text {reachable }}-\left\{v_{y(s)}\right\}\right) \cup V_{I}^{\prime} \cup V_{I I}^{\prime}, \text { where } \\
& V_{I}^{\prime}=\left(\left\{v_{y(s)}^{\prime}\right\} \cup V_{v_{y(s)}^{\prime}}^{\text {reachable }}\right) \text { and } V_{I I}^{\prime}=\left(\left\{v_{y\left(s^{*}\right)}^{\prime}\right\} \cup V_{v_{y\left(s^{*}\right)}^{\prime}}^{\text {reachable }}\right) \\
& E^{\prime}=\left(E-E_{v_{y(s)}}^{\text {reachable }}-E_{v_{y(s)}}^{\text {in }}\right) \cup E_{I}^{\prime} \cup E_{I I}^{\prime}, \text { where }
\end{aligned}
$$




$$
\begin{aligned}
& E_{I}^{\prime}=\left(E_{v_{y(s)}^{\prime}}^{\text {reachable }}-E_{v_{y}^{\prime} \sim \operatorname{tail}\left(e_{r}\right)}^{i n f\left(v_{y(s)}^{\prime} \text { tail }\left(e_{r}\right)\right)}\right) \cup E_{v_{y(s)}^{\prime}}^{i n(N T)} \cup \\
& \left(E_{v_{y(s)}^{\prime}(T)}^{i n(T)}-E_{y_{y^{\prime}(s)}}^{\operatorname{conf}\left(e_{x} \in E_{v_{y(s)}^{\prime}}^{i n}, e_{r}\right)}\right) \text { and } \\
& E_{I I}^{\prime}=\left(E_{v_{y\left(s^{*}\right)}^{\prime}}^{\text {reachable }}-E^{\text {conf }\left(e_{x} \in E_{e_{k} \sim e_{r}}^{\text {out }}, e_{r}\right)}\right) \cup E_{v_{y\left(s^{*}\right)}^{\prime}}^{\text {in }} \cup\left(\left\{e_{k}\right\} \cup E_{v_{w\left(s^{*}\right)}^{\prime}}^{\text {depend }}\right)
\end{aligned}
$$

Due to the removal of edges with infeasible conditions from $G^{\prime}\left(V^{\prime}, E^{\prime}\right)$, unreachable subgraphs may result. A simple DF graph traversal can be used to eliminate unreachable subgraphs from $G^{\prime}\left(V^{\prime}, E^{\prime}\right)$. Furthermore, if the condition of an edge $e_{i}$ cannot be satisfied due to the actions of another edge $e_{j}$, it is removed from the graph during the condition inconsistency analysis as described next.

3.2.2 Condition Inconsistencies. In this section, the detection and elimination of condition inconsistencies (if any) is considered, which is the next step after the action inconsistencies are eliminated from the EFSM model.

A test sequence generated from an EFSM should avoid including two or more edges with conflicting conditions. Since the conditions of the edges of a test sequence constitute a system of constraints, algorithms available for solving linear programming problems can be used in deciding whether a certain path predicate is feasible [1].

The edge conditions in a path from the starting node $v_{0}$ to a node $v_{i}$ can be represented in matrices. A triplet of matrices are defined as $C(m \times p), \tilde{O P}$ $(p \times 1)$, and $\tilde{D}(p \times 1)$, where $m$ is the number of variables, $p$ is the number of conditions in the path from $v_{0}$ to $v_{i}, C$ is the coefficient matrix, $\tilde{O P}$ is the operator vector containing the relations of $=,<,\rangle, \neq \cdots$, etc., and $\tilde{D}$ is the scalar vector containing the scalar values of the conditions in the path.

The AUM pairs discussed in Section 3.2.1 are applied to the edge conditions of the EFSM graph as follows. A single condition of an edge $e_{r}=\left(v_{i}, v_{j}\right)$ is in the form of $\tilde{C} * \tilde{V}(\tilde{O P}) \tilde{D}$. The condition of $e_{r}$ will be modified based on the symbolic values of the variables $v_{a} r_{0}$ through $v_{m-1}$, which are represented by the $\operatorname{AUM}\left(v_{i}, J\right)$. The current values of the variables including all the modifications represented by an AUM pair of $v_{i}$ are in the form of: $\tilde{V}=$ $A_{v_{i}, k} * \tilde{V}+\tilde{B}_{v_{i}, k}$. Substituting $\tilde{V}$ values in an edge condition will result in $\tilde{C}\left(A_{v_{i}, k} * \tilde{V}+\tilde{B}_{v_{i}, k}\right)(\tilde{O P}) \tilde{D}$, which simplifies as $\tilde{E} * \tilde{V}(\tilde{O P}) f$, where $\tilde{E}=\tilde{C} * A_{v_{i}, k}$ is an $m$-element vector and $f$ is a scalar. An edge $e_{r}=\left(v_{i}, v_{j}\right)$ whose condition is infeasible based on the AUM pairs of $v_{i}$ is deleted from the graph. The values assumed by the variables used in the condition of $e_{r}$ can be determined from:

$$
C * \tilde{V}=C *\left(A_{v_{i}, k} * \tilde{V}+\tilde{B}_{v_{i}, k}\right)
$$


where $C$ is the coefficient matrix for the condition of $e_{r}$ and $0 \leq k<J$, where $J$ is the number of AUM pairs associated with $v_{i}$.

The accumulated different conditions of the paths leading to $v_{i}$ can be represented by a set of Accumulated Condition Matrix (ACM) triplets: $\operatorname{ACM}\left(v_{i}, J\right)$ $=\left(C_{v_{i}, 0}, \quad \tilde{O P} v_{v_{i}, 0}, \quad \tilde{D}_{v_{i}, 0}, C_{v_{i}, 1}, \tilde{O P}_{v_{i}, 1}, \quad \tilde{D}_{v_{i}, 1}, \cdots, \quad C_{v_{i}, J-1}, \tilde{O P} v_{v_{i}, J-1}\right.$, $\left.\tilde{D}_{v_{i}, J-1}\right)$, where $C_{v_{i}, k}, O P_{v_{i}, k}, \tilde{D}_{v_{i}, k}$, and $J$ are the $k^{\text {th }}$ coefficient matrix, $k^{\text {th }}$ operator matrix, $k^{\text {th }}$ scalar value matrix $(0 \leq k<J)$, and the number of the ACM triplets associated with $v_{i}$, respectively.

3.2.2.1 Detection of Condition Inconsistencies. The condition inconsistency detection is performed by traversing the graph in a depth-first (DF) manner. Condition inconsistencies can be detected and eliminated by focusing on the ACM triplets of a node $v_{y}, \operatorname{ACM}\left(v_{y}, J\right)$, one triplet at a time. Let $\operatorname{ACM}\left(e_{r}\right)$ be the triplet representing the condition of $e_{r} \in E_{v_{y}}^{\text {reachable }}$. Furthermore, let $V A R_{e_{i}}^{\text {con-used }} \in V A R$ be the set of variables used in the conditions of $e_{i} \in E$. Once a node $v_{y}$ is visited by traversing an edge $e_{k}=\left(v_{x}, v_{y}\right)$, each edge $e_{r}=\left(v_{w}, v_{z}\right)$, where $v_{w} \in V_{v_{y}}^{\text {reachable }}$, such that

$$
V A R_{e_{r}}^{\text {con-used }} \cap V A R_{e_{k}}^{\text {con-used }} \neq \emptyset
$$

is identified. The consistency between the conditions of $e_{k}$ and $e_{r}$ is then checked by concatenating $\operatorname{ACM}\left(e_{r}\right)$ to the $\operatorname{ACM}\left(v_{y}, p\right)$, where $0 \leq p<J$.

Let us introduce the following definition:

$\operatorname{Feas}\left(\operatorname{ACM}\left(v_{y}, p\right), e_{r}\right)= \begin{cases}1, & \text { if } \operatorname{ACM}\left(v_{y}, p\right) \# \operatorname{ACM}\left(e_{r}\right) \text { has a solution } \\ 0, & \text { otherwise }\end{cases}$ where \# denotes concatenation of the two ACM triplets.

If the new constraints formed by $\operatorname{ACM}\left(v_{y}, p\right) \# \operatorname{ACM}\left(e_{r}\right)$ has a solution, $e_{r}$ is said to be consistent with the edges whose conditions constitute $\operatorname{ACM}\left(v_{y}, p\right)$. Otherwise $e_{r}$ is inconsistent with the edges whose conditions constitute $\operatorname{ACM}\left(v_{y}, p\right)$. The algorithm in Figure 3 stops when either a condition inconsistency is found or the DF graph traversal is completed.

3.2.2.2 Elimination of Condition Inconsistencies. A condition inconsistency between two edges $e_{k}=\left(v_{x}, v_{y}\right)$ and $e_{r}=\left(v_{w}, v_{z}\right)$ is eliminated by splitting the nodes $\left(\left\{v_{y}\right\} \cup\left\{v_{i} \in V_{\text {tail }\left(v_{y}\right)}^{\text {reachable }}: v_{i} \sim v_{w}\right\}\right)$, with their incoming and outgoing edges, into two subgraphs such that each subgraph contains either $e_{k}$ or $e_{r}$, but not both. The outgoing edges of the nodes $\left\{v_{i} \in V_{\text {tail }\left(v_{y}\right)}^{\text {reache }}: v_{i} \leadsto v_{w}\right\}$ will be referred to as $E_{v_{y} \leadsto v_{w}}^{\text {out }}$. The condition inconsistency elimination algorithm is given in Figure 4.

Since the consistency of the edges of $E_{v_{y}}^{i n(N T)}$ with $E_{v_{w}}^{o u t}$ cannot be decided during the graph splitting, copies of these edges are included temporarily in the 


\section{Condition Inconsistency Detection}

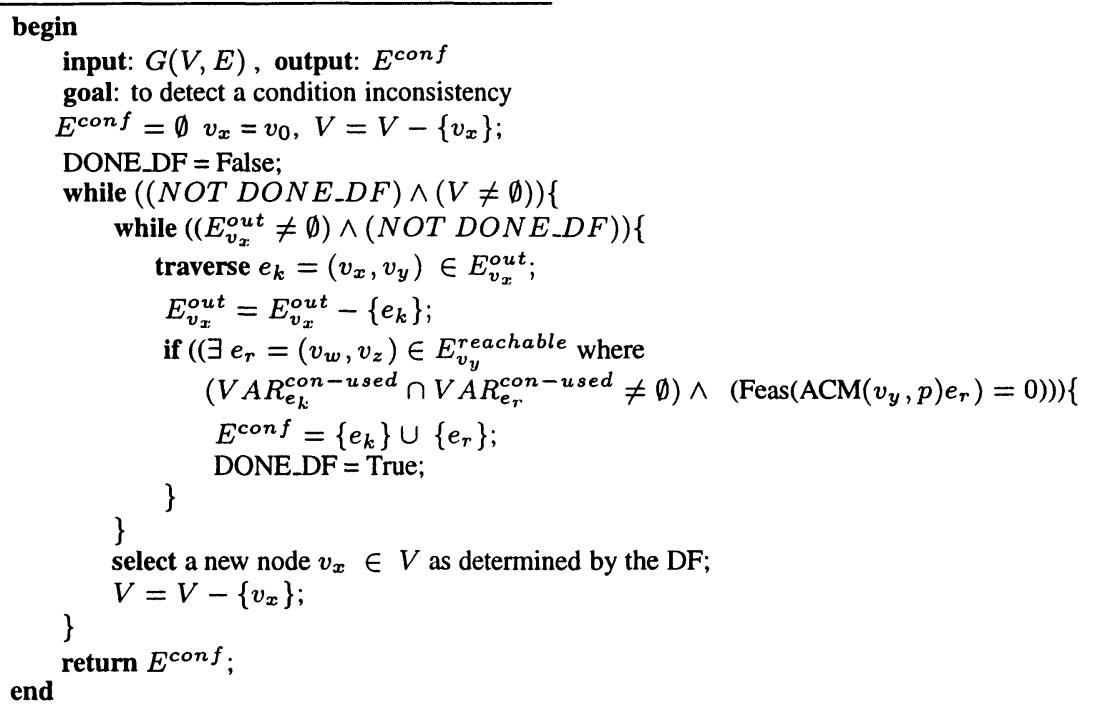

Figure 3. Condition inconsistency detection algorithm.

same subgraph with $e_{k}=\left(v_{x}, v_{y}\right)$. The influence of $E_{v_{w}}^{\text {depend }}$ on the conditions of the outgoing edges of $v_{w} \in V_{\text {tail }\left(e_{k}\right)}^{\text {reachable }}$ must be analyzed later when each edge in $E_{v_{w}}^{\text {depend }}$ is traversed, where $E_{v_{w}}^{\text {depend }}$ is the set of edges which are temporarily included in the subgraph containing $e_{k}$. An edge $e_{i\left(s^{*}\right)}^{\prime} \in E_{v_{w^{\prime}\left(s^{*}\right)}^{d e p e n d}}^{E^{\prime}}$ whose condition is found to be inconsistent with the condition of an outgoing edge of $v_{w(s)}^{\prime}$ is removed from the graph.

After a condition inconsistency is detected and eliminated, the resulting graph $G^{\prime}\left(V^{\prime}, E^{\prime}\right)$ is defined as:

$$
\begin{gathered}
V^{\prime}=\left(V-\left\{v_{i} \mid v_{i} \in V_{v_{y(s)}}^{\text {reachable }} \wedge v_{i} \leadsto v_{w}\right\}-\left\{v_{y(s)}\right\}\right) \cup V_{I}^{\prime} \cup V_{I I}^{\prime} \\
\text { where } V_{I}^{\prime}=\left(\left\{v_{y(s)}^{\prime}\right\} \cup V_{v_{y(s)}^{\prime}}^{\text {reachable }}\right) \text { and } V_{I I}^{\prime}=\left(\left\{v_{y\left(s^{*}\right)}^{\prime}\right\} \cup V_{v_{y\left(s^{*}\right)}^{\prime}}^{\text {reachable }}\right) \\
E^{\prime}=\left(E-E_{v_{y} \sim v_{w}}^{\text {out }}-E_{v_{y(s)}}^{\text {in }}\right) \cup E_{I}^{\prime} \cup E_{I I}^{\prime} \text {, where } \\
\qquad E_{I}^{\prime}=E_{v_{y(s)}^{\prime}}^{i n(N T)} \cup E_{v_{y(s)}^{\prime}}^{\text {in }(T)} \cup E_{v_{y(s)}^{\prime}}^{\text {reachable and }}
\end{gathered}
$$




\section{Condition Inconsistency Elimination}

input: $e_{k}=\left(v_{x}, v_{y}\right)$ and $e_{r}=\left(v_{w}, v_{z}\right)$, where a condition inconsistency exists between $e_{k}$ and $e_{r}$

goal: to eliminate the inconsistency between $e_{k}$ and $e_{r}$ by creating two begin

subgraphs such that each subgraph contains either $e_{k}$ or $e_{r}$, but not both

Split $v_{y(s)}$ into two nodes as: $v_{y(s)}^{\prime}$ and $v_{y\left(s^{*}\right)}^{\prime}$;

Split $\forall v_{i(s)} \in V_{v_{y(s)}^{r e a c h a b l e}}^{\text {re }} v_{i} \sim v_{w}$ into two nodes as:

$v_{i(s)}^{\prime} \in V_{v_{y(s)}^{\prime}}^{\text {reachable }}$ and $v_{i\left(s^{*}\right)}^{\prime} \in V_{v_{y\left(s^{*}\right)}^{\text {reachable }}}^{\text {reach }}$

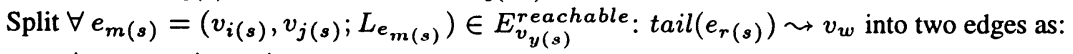

$e_{m(s)}^{\prime}=\left(v_{i(s)}^{\prime}, v_{j(s)}^{\prime} ; L_{e_{m(s)}}\right) \in E_{v_{y(s)}^{\prime}}^{\text {reachable }}$ and

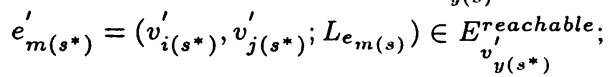

Split $\forall e_{m(s)}=\left(v_{i(s)}, v_{y(s)} ; L_{e_{m(s)}}\right) \in\left(E_{v_{y(s)}^{i n(T)}}^{i n}-\left\{e_{k}\right\}\right)$ into two edges as:

$e_{m(s)}^{\prime}=\left(v_{i(s)}, v_{y(s)}^{\prime} ; L_{e_{m(s)}}\right) \in E_{v_{y(s)}^{\prime}}^{i n(T)}$ and

$e_{m\left(s^{*}\right)}^{\prime}=\left(v_{i(s)}, v_{y\left(s^{*}\right)}^{\prime} ; L_{e_{m(s)}}\right) \in E_{v_{w\left(s^{*}\right)}^{i n}}^{\prime}$ where $v_{w(s)}$ is the node whose outgoing

edges are inconsistent with $e_{k(s)}$;

Split $\forall e_{m(s)}=\left(v_{i(s)}, v_{y(s)} ; L_{e_{m(s)}}\right) \in E_{v_{y(s)}}^{i n(N T)}$ into two edges as:

$$
\begin{aligned}
& e_{m(s)}^{\prime}=\left(v_{i(s)}, v_{y(s)}^{\prime}, L_{e_{m(s)}}\right) \in E_{v_{y(s)}^{i n(N T)} \text { and }}^{\text {ing }} \\
& e_{m\left(s^{*}\right)}^{\prime}=\left(v_{i(s)}, v_{y\left(s^{*}\right)}^{\prime}, L_{e_{m(s)}}\right) \in E_{v_{w\left(s^{*}\right)}^{\text {depend }}}^{\prime} ;
\end{aligned}
$$

end

Figure 4. Condition inconsistency elimination algorithm.

$$
\begin{gathered}
E_{I I}^{\prime}=E_{v_{w\left(s^{*}\right)}^{\text {depend }}}^{\prime} \cup E_{v_{y\left(s^{*}\right)}^{\prime}(T)}^{i n(T)} \cup\left\{e_{k}\right\} \cup\left(E_{v_{y\left(s^{*}\right)}^{\prime}}^{\text {reachable }}-\right. \\
\left.\left\{e_{i} \in E_{v_{y\left(s^{*}\right)}^{r e a c h a b l e}}^{\text {reable }}: \operatorname{Feas}\left(\operatorname{ACM}\left(v_{y}, p\right) \# \operatorname{ACM}\left(e_{i}\right)\right)=0\right\}\right)
\end{gathered}
$$

As for the action inconsistency detection and removal case, the algorithms restart after each graph split.

\subsection{The Complexity of the Algorithms}

The complexity of the action inconsistency detection and elimination is contributed by a two-phase MBF graph traversal, constructing the number of AUM pairs for each node, and executing the linear programming for each edge for each AUM pair.

The complexity for the two-phase MBF graph traversal is $\left(O\left(E^{2}\right)\right.$. For each node $v_{i}$, the number of AUM pairs is $\sum_{1}^{|V|-1}\left|E^{v_{j} \rightarrow v_{i}}\right| \times\left|\operatorname{AUM}\left(v_{j}, J\right)\right|$ (where $\left|E^{v_{j} \rightarrow v_{i}}\right|$ is the number of edges from $v_{j}$ to $\left.v_{i}\right)$ such that $\exists e_{k}=\left(v_{j}, v_{i}\right)$. Linear programming takes $\min \left(m^{2}, S^{2}\right)$ steps where $m$ is the number of variables and $S$ is the number of constraints [1]. 


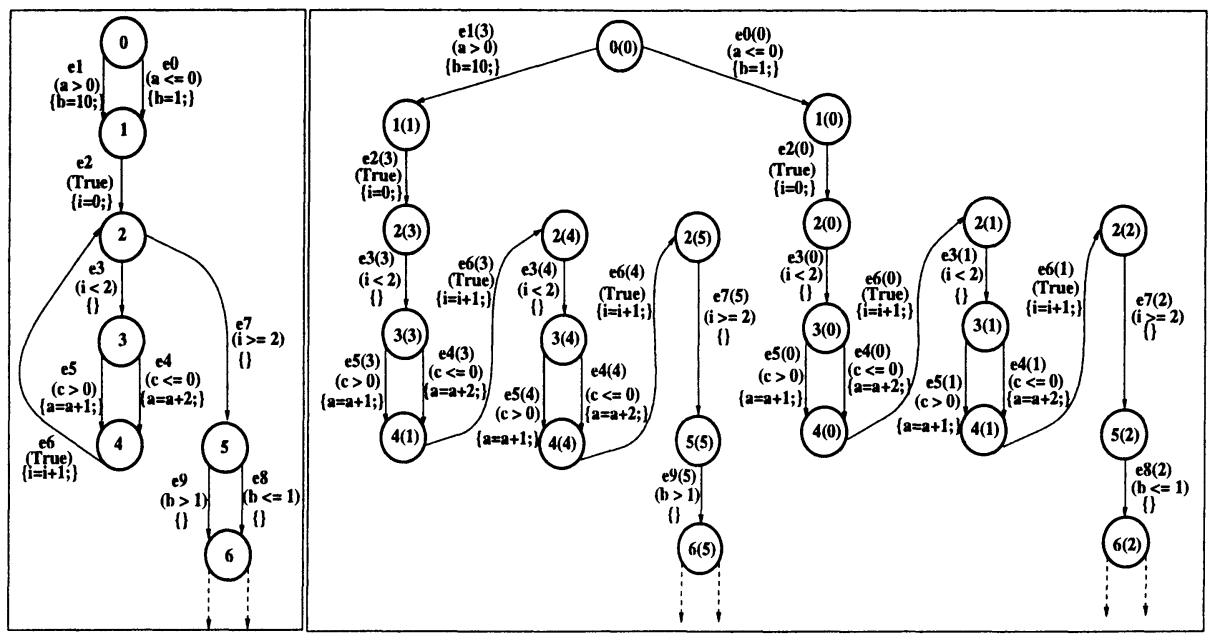

Figure 5. a) An EFSM graph

b) The resulting EFSM graph after the action inconsistencies are eliminated from the EFSM graph shown in Figure 5.a.

The complexity for the condition inconsistency detection and elimination is bounded by the number of times the graph is split. Therefore, for the general case, the complexity of algorithms for handling the action inconsistencies is exponential with respect to the number of simple paths (i.e., the number AUM pairs). Similarly, the condition inconsistency elimination can be exponential with respect to the number of graph splits. However, based on our experience with several protocols (even with nested and/or concatenated loops), the complexity of both algorithms and, hence, the size of the consistent graph are bounded by the number of different values each condition variable assumes.

\section{EXAMPLE}

In the EFSM graphs in all the figures, the condition and actions of an edge are inclosed in parentheses " $(\cdots)$ " and curly braces " $\{\cdots\}$ ", respectively.

In Figure 5 the $\operatorname{AUM}\left(v_{1}, J\right)$ associated with $v_{1}$ is based on the $\operatorname{AUM}\left(v_{0}, 1\right)$ and the actions of the edges between $v_{0}$ and $v_{1}$ (i.e., $e_{0}$ and $e_{1}$ ). The first AUM pair of $v_{1}\left(A_{v_{1}, 0}, \tilde{B}_{v_{1}, 0}\right)$

$$
A_{v_{1}, 0}=\left[\begin{array}{lllll}
1 & 0 & 0 & 0 & 0 \\
0 & 0 & 0 & 0 & 0 \\
0 & 0 & 1 & 0 & 0 \\
0 & 0 & 0 & 1 & 0 \\
0 & 0 & 0 & 0 & 1
\end{array}\right] \quad \tilde{B}_{v_{1}, 0}=\left[\begin{array}{l}
0 \\
1 \\
0 \\
0 \\
0
\end{array}\right]
$$




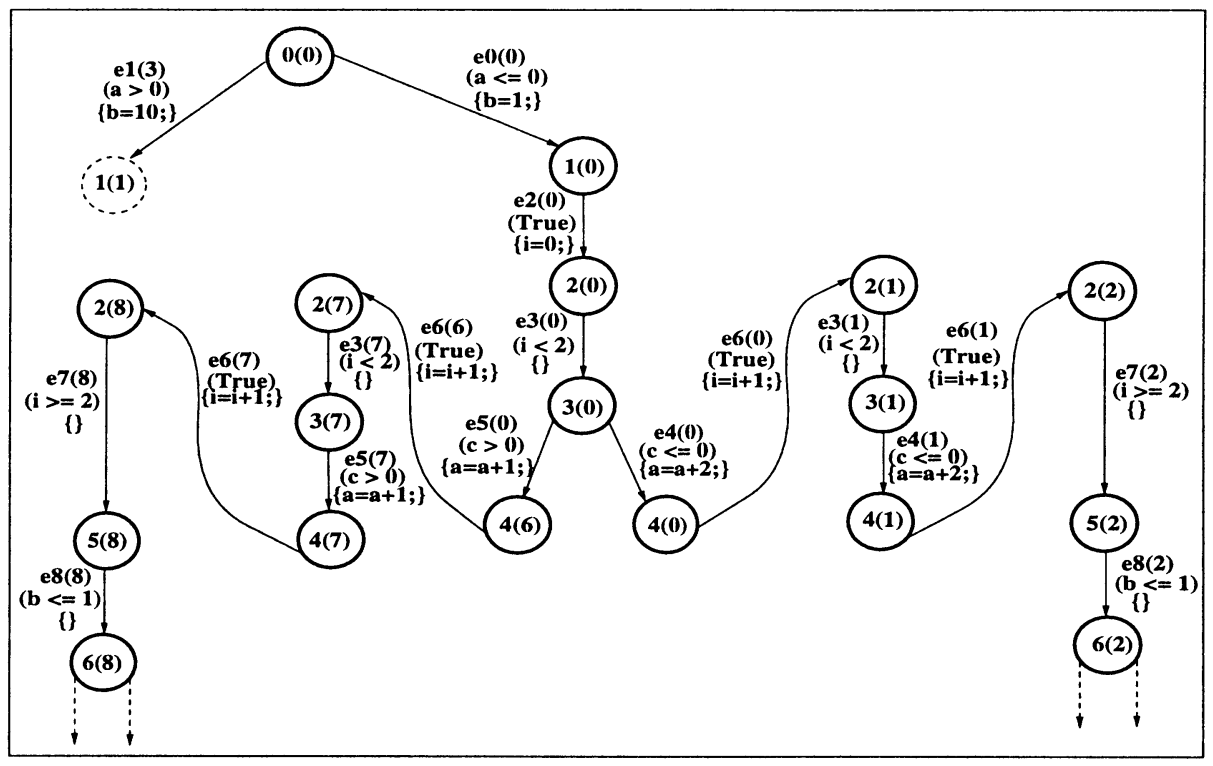

Figure 6. The EFSM graph after the graph of Figure 5.b is split due to the condition of $e_{4(0)}$ (the subgraph starting from node $v_{1(0)}$ is shown).

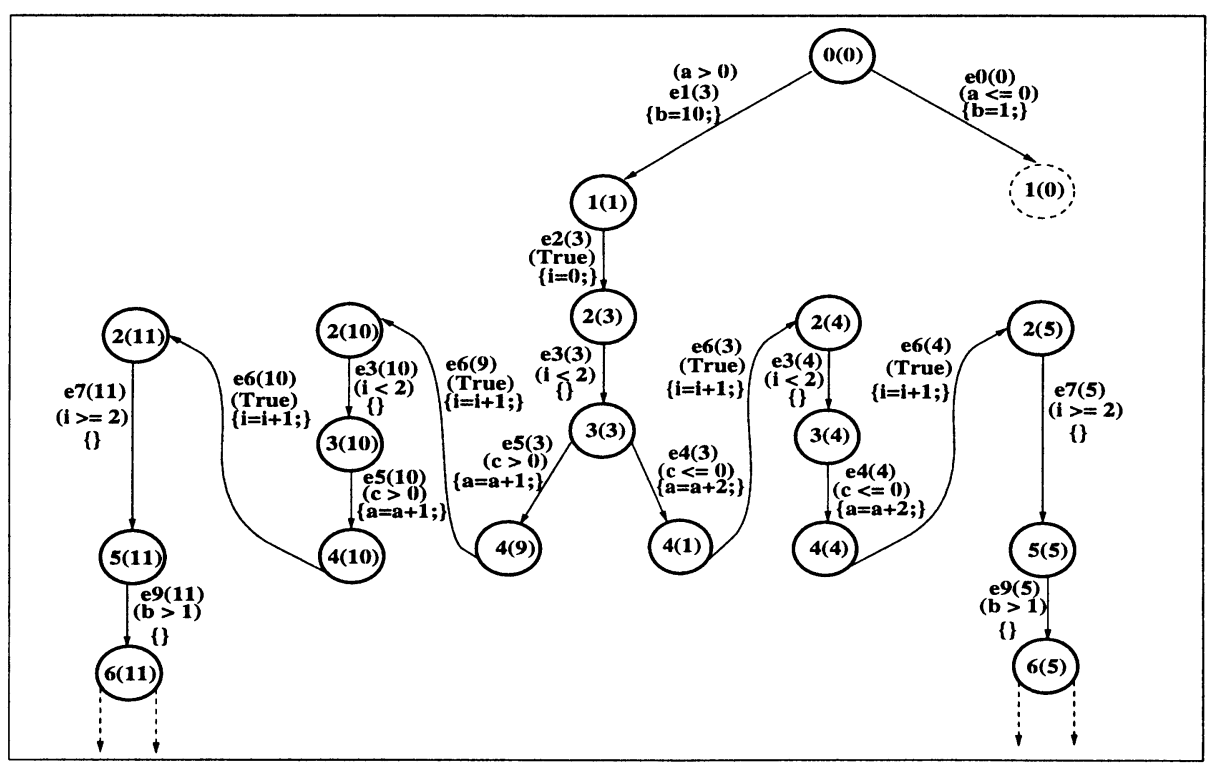

Figure 7. The EFSM graph after the graph of Figure 6 is split due to the condition of $e_{4(3)}$ (the subgraph starting from the node $v_{1(0)}$ is identical to the subgraph starting from $v_{1(0)}$ of Figure 6). 
is formed when $e_{0}$ is traversed. The values assumed by $a, b, c, i$, and True after $e_{0}$ is traversed can be determined from:

$$
\tilde{V}=A_{v_{1}, 0} * \tilde{V}+\tilde{B}
$$

where $\tilde{V}(m \times 1)$ is the vector for the variables. Equation (2) yields $a=a, b=1$, $c=c, i=i$, and True $=$ True. Similarly, due to the action associated with $e_{1}$, a new AUM pair $\left(A_{v_{1}, 1}, \tilde{B}_{v_{1}, 1}\right)$, where $b=10$, is formed for $v_{1}$ when $e_{1}$ is traversed. P1-MBF continues until the loop is advanced two iterations as shown in Figure 5b. The P2-MBF graph traversal is invoked upon visiting $v_{1}$ of Figure 5b. P2-MBF proceeds until $v_{5(2)}$ is visited. An action inconsistency is detected between the edges leading to $v_{1(0)}$ and the outgoing edges of $v_{5(2)}$. As a result, the graph of Figure $5 \mathrm{~b}$ is split as shown in Figure 5.b. There are no action inconsistencies in the EFSM graph of Figure 5.b. The DF graph traversal of Figure 5.b proceeds with the edges of $e_{2(0)}, e_{3(0)}, e_{4(0)}, \cdots$. The $\mathrm{ACM}$ triplet resulting from the traversal of the path $e_{0(0)} \cdot e_{2(0)} \cdot e_{3(0)} \cdot e_{4(0)}$ is:

$$
\left[\begin{array}{lllll}
1 & 0 & 0 & 0 & 0 \\
0 & 0 & 0 & 0 & 1 \\
0 & 0 & 0 & 1 & 0 \\
0 & 0 & 1 & 0 & 0
\end{array}\right] \quad\left[\begin{array}{l}
\leqq \\
\leq \\
\leq
\end{array}\right]\left[\begin{array}{l}
0 \\
1 \\
2 \\
0
\end{array}\right]
$$

It is found that the edges $e_{4(1)}$ and $e_{5(1)}$ use the variable $c$ in their conditions, which is also used in the condition of $e_{4(0)}$. Since the $\operatorname{ACM}\left(v_{4(0)}, 1\right) \#$ $\operatorname{ACM}\left(e_{5(1)}\right)$ system of constraints does not have a solution, there is a condition inconsistency between $e_{4(0)}$ and $e_{5(1)}$. To eliminate this condition inconsistency, the graph of Figure 5.b is split such that $e_{4(0)}$ and $e_{5(1)}$ are placed into two separate subgraphs as shown in Figure 6.

Similarly, when $e_{4(3)}$ of the graph of Figure 6 is traversed, the system of constraints formed by $\operatorname{ACM}\left(v_{4(3)}, 1\right) \# \operatorname{ACM}\left(e_{5(4)}\right)$ has no feasible solution. As a result, the EFSM graph of Figure 6 is split. The graph of Figure 7 contains no inconsistencies.

\section{CONCLUSIONS}

The method presented in this paper enables the generation of realizable test sequences from a class of EFSMs. Due to the variable interdependencies among the actions and conditions, certain paths of an EFSM model may not be feasible. Algorithms to eliminate these inconsistencies from a class of EFSMs to produce consistent EFSMs are presented. The elimination of inconsistencies is achieved by using a graph splitting technique based on symbolic execution and linear programming. To avoid unnecessary state explosion, new nodes and edges are created only when needed during the inconsistency elimination. Although the well-known state explosion cannot be avoided for all EFSMs, the growth of the state space is limited. For the cases where the state explosion is 
unavoidable, the size of the new graph is constantly monitored as the algorithms eliminate the inconsistencies.

Since consistent EFSMs behave like FSMs, the FSM-based test generation techniques can be used to generate feasible test sequences from the consistent EFSMs.

Currently this methodology is being applied to generate tests for the MILSTD 188-220 protocol [7].

\section{References}

[1] I. Adler and N. Megiddo, "A Simplex Algorithm Whose Average Number of Steps is Bound Between Two Quadratic Functions of the Smaller Dimension," Journal of the $A C M$, vol. 32, No. 4, Oct. 1985, pp. 871-895.

[2] E. Brinksma, “A Theory for the Derivation of Tests," In Proc. IFIP Protocol Specif. Test. Verif. (PSTV), Amsterdam: North Holland, 1988.

[3] S. Chanson and J. Zhu, "A Unified Approach to Protocol Test Sequence Generation," Proc. of IEEE INFOCOM 1993, pp. 1d.1.1-1d.1.9.

[4] K. T. Cheng and A. S. Krishnakumar, "Automated Generation of Functional Vectors Using the Extended Finite State Machine Model", ACM Trans. on Design Automation, vol. 1, No. 1, Jan. 1996, pp. 57-79.

[5] P. D. Coward, “Symbolic Execution Systems - a Review,” Software Eng. Jour., Nov. 1988, pp. 229-239.

[6] A. En-Nauaary, R. Dssaouli, F. Khendek, and A. Elqortobi, "Timed Test Cases Generation Based on State Characterisation Technique," Proc. IEEE Real-Time Syst. Symp. (RTSS), pp. 220-229, Madrid, Spain, Dec. 1998.

[7] M. Fecko, U. Uyar, A. Duale, P. Amer, "Test Generation in the Presence of Conflicting Timers," The IFIP 13th International Conference on Testing of Communicating Systems, Ottawa, Canada, 2000.

[8] M. Fecko, U. Uyar, P. Amer, A. Sethi, T. Dzik, R. Menell, and M. McMahon, "A Success Story of Formal Description Techniques: Estelle Specification and Test Generation for MIL-STD 188-220," In R. Lai, ed, FDT in Practice, vol. 23 of Comput. Communic. (special issue), Spring 2000 (in press).

[9] S. Fujiwara, G. Bochmann, F. Khendek, M. Amolou, and A. Ghedamsi, "Test Selection Based on Finite State Machine Models," IEEE Trans. on Software Engr., 17(6):591-603, June 1991.

[10] T. Higashino, A. Nakata, K. Taniguchi, and A. R. Cavalli, "Generating Test Cases for a Timed I/O Automaton Model," In Proc. IFIP Int'l Workshop on Test Commun. Syst. (IWTCS), pp. 197-214, Budapest, Hungary, Sept. 1999.

[11] D. Hogrefe, "On the Development of a Standard for Conformance Testing Based on Formal Specifications," Comput. Stand. Interf., 14(3):185-190, 1992. Test Cases from SDL Specifications," SDL '89: The Language at Work, Proc. Fourth SDL Forum, pp. 267-279, 1989.

[12] X. Li, T. Higashino, M. Higuchi, and K. Taniguchi, "Automatic Generation of Extended UIO Sequences for Communication Protocols in an EFSM Model," In Proc. of 7th Int' 1 Workshop on Protocol Test Systems, Tokyo, Japan, Nov. 1997, pp. 225-240. 
[13] G. Luo, G. Bochmann, and A. F. Petrenko, "Test Selection Based on Communicating Non-deterministic Finite State Machines Using a Generalized Wp-Method," IEEE Trans. on Software Engr., 20(2):149-162, 1994.

[14] R. Miller and S. Paul, "Generating Conformance Test Sequences for Combined Control Flow and Data Flow of Communication Protocols," In Proc. of 12th International Symposium of Protocol Specification, Testing, and Verification, 1992, pp. 12-27.

[15] K. Salah, H. Ural, and A. Williams, "Test Generation Based on Control and Data Dependencies within Systems Specified in SDL," (to appear in Computer Communications).

[16] B. Sarikaya, G. Bochmann, and E. Cerny, "A Test Design Methodology for Protocol Testing," IEEE Trans. on Software Eng., Vol. SE-13, No. 5, May 1987, pp. 518-531.

[17] J. Tretmans, "Conformance Testing with Labelled Transitions Systems and Test Generation," Comput. Networks ISDN Syst., 29(1):49-79, 1996.

[18] H. Ural, "Formal Methods for Test Sequence Generation," IEEE Trans. on Commun., 39(4):514-523, 1992.

[19] H. Ural, "A Test Derivation Method for Protocol Conformance Testing," Proc. Sixth Int' Conf. Protocol Specification, Testing and Verification, 1989, pp. 347-358.

[20] H. Ural and B. Yang, "A Test Sequence Selection Method for Protocol Testing," IEEE Trans. on Communications, vol., 39, No., 4, Apr. 1991, pp. 514-523.

[21] M. U. Uyar and A. Y. Duale, "Resolving Inconsistencies in VHDL Specifications," In Proc. IEEE Milit. Commun. Conf. (MILCOM), Atlantic City, NJ., Oct. 1999 , No. 5.1.3.

[22] M. U. Uyar and A. Y. Duale, "Modeling VHDL Specifications as Consistent EFSMs," In Proc. IEEE Milit. Commun. Conf. (MILCOM), Monterey, CA., Oct. 1997, pp. 740-744. 